\title{
Natural and Projectively Invariant Quantizations on Supermanifolds
}

\author{
Thomas LEUTHER and Fabian RADOUX
}

Institute of Mathematics, Grande Traverse 12, B-4000 Liège, Belgium

E-mail: thomas.leuther@ulg.ac.be,fabian.radoux@ulg.ac.be

Received October 05, 2010, in final form March 23, 2011; Published online March 31, 2011

doi:10.3842/SIGMA.2011.034

\begin{abstract}
The existence of a natural and projectively invariant quantization in the sense of P. Lecomte [Progr. Theoret. Phys. Suppl. (2001), no. 144, 125-132] was proved by M. Bordemann [math.DG/0208171], using the framework of Thomas-Whitehead connections. We extend the problem to the context of supermanifolds and adapt M. Bordemann's method in order to solve it. The obtained quantization appears as the natural globalization of the $\mathfrak{p g l}(n+1 \mid m)$-equivariant quantization on $\mathbb{R}^{n \mid m}$ constructed by P. Mathonet and F. Radoux in [arXiv:1003.3320]. Our quantization is also a prolongation to arbitrary degree symbols of the projectively invariant quantization constructed by J. George in [arXiv:0909.5419] for symbols of degree two.
\end{abstract}

Key words: supergeometry; differential operators; projective invariance; quantization maps

2010 Mathematics Subject Classification: 53B05; 53B10; 53D50; 58A50

\section{Introduction}

The quantization is a concept that comes from physics. The quantization of a classical system whose phase space is a symplectic manifold $(\mathcal{M}, \omega)$, consists in the construction of a Hilbert space $H$ and a correspondence between classical and quantum observables. Classical observables are smooth functions on $\mathcal{M}$ while quantum observables are self-adjoint operators on $H$. The concept of quantization has been formulated by P. Dirac [5], while trigged by the similarity between the formalisms of classical and quantum mechanics. At the beginning, the problem of the quantization consisted in finding a linear bijection $Q$ between $C^{\infty}(\mathcal{M})$ and a space of operators on $H$ verifying three properties: the bijection $Q$ has to transform the constant function 1 into the identity operator, the conjugation into the adjunction and the Poisson bracket into the commutator.

Prequantization [7], which associates with a function on $\mathcal{M}$ a differential operator on the Hilbert space of complex functions square-integrable on $\mathcal{M}$, gives a positive answer to the Dirac problem. However, even when the phase space $\mathcal{M}$ is the cotangent bundle of a certain manifold $M$, it is not satisfactory because it gives us the Hilbert space $L^{2}\left(T^{*} M\right)$ which is too large for a physically reasonable quantum system (it contains wave functions depending both on the position and the momentum coordinates, see [16, Chapter 5] for details). Geometric quantization [16], however, fixes this issue by means of a polarization: if $\mathcal{M}=T^{*} M$, the vertical polarization allows of reducing the Hilbert space $L^{2}\left(T^{*} M\right)$ to the space $L^{2}(M)$. Geometric quantization is then applied only to a subset of observables, those preserving the chosen polarization.

A priori, geometric quantization can be extended to the whole set of observables in many different ways. One can ask whether such an extension is unique if one requires extra conditions on the quantization map. 
The uniqueness of a quantization procedure is often linked to a symmetry group. A vector field $X$ on a manifold $M$ can be lifted in a natural way to a vector field on $T^{*} M$, thereby defining an action of the algebra of vector fields on $M, \operatorname{Vect}(M)$, on the space of functions on $T^{*} M$ polynomial in the fibers, called the space of symbols. It turns out that geometric quantization is the unique $\operatorname{Vect}(M)$-equivariant map from the space of symbols of degree less than or equal to one to the space of differential operators on $M$, up to a normalization. However, geometric quantization cannot be extended to the whole space of symbols if one requires equivariance with respect to the Lie algebra $\operatorname{Vect}(M)$, due to cohomological reasons [11]. One can ask whether there exists a Lie subalgebra $\mathfrak{g} \subset \operatorname{Vect}(M)$ for which the quantization map is $\mathfrak{g}$-equivariant. This $\mathfrak{g}$ is supposed to be "big enough" to attain the uniqueness, but "small enough" to acquire the extension of the geometric quantization to the whole space of symbols.

When $M=\mathbb{R}^{n}$ with a $P G L(n+1, \mathbb{R})$-structure, the quantization map has been investigated by P. Lecomte and V. Ovsienko in [10]. They showed that there exists a unique quantization map that is $\mathfrak{p g l}(n+1, \mathbb{R})$-equivariant.

The concept of $\mathfrak{p g l}(n+1, \mathbb{R})$-equivariant quantization on $\mathbb{R}^{n}$ has a counterpart on an arbitrary manifold $M$ [12]. It aims at constructing a quantization map by means of a connection, depending only on its projective class (i.e. projectively invariant) and natural in all of its arguments. This quantization coincides with the projectively equivariant quantization when $M=\mathbb{R}^{n}$ and the connection is the projectively flat one.

The existence of such a quantization procedure was proved by M. Bordemann [1]. With each class of torsion-free connections, he associated a unique linear connection, $\tilde{\nabla}$, on a principal line bundle $\tilde{M} \rightarrow M$. He managed to lift the space of symbols in a natural way to suitable tensors on $\tilde{M}$. He then applied the so-called standard ordering to construct the projectively invariant quantization map.

Recently, P. Mathonet and F. Radoux [15] constructed a super-version of the $\mathfrak{p g l}(n+1, \mathbb{R})$ equivariant quantization on $\mathbb{R}^{n}$. This super-quantization is a quantization on the superspace $\mathbb{R}^{n \mid m}$ which is equivariant with respect to a Lie superalgebra of supervector fields on $\mathbb{R}^{n \mid m}$, this Lie superalgebra being isomorphic to $\mathfrak{p g l}(n+1 \mid m)$.

In the same way as in the classical case, one can wonder if this super-quantization can be globalized. A partial positive answer to this question has been given in [6], where a projectively invariant quantization on supermanifolds has been constructed for symbols of degree less than or equal to two.

In this paper we prove the existence of a projectively invariant and natural quantization on a supermanifold. In Section 2, we recall the fundamental tools which we will need in the sequel (tensor densities, differential operators, connections) and we give the definition of a natural projectively invariant quantization. Next, we solve the problem of existence of such a quantization by adapting Bordemann's method. To do this, we use the "Thomas connection" constructed by J. George [6], this connection being the super-version of the Thomas connection used by M. Bordemann [1].

\section{Problem setting}

For the sake of completeness, we briefly recall in this section the definitions of tensor densities, differential operators and symbols on supermanifolds. Then we set the problem of the existence of natural and projectively invariant quantizations on supermanifolds. Throughout this paper, we denote by $M$ a smooth supermanifold of dimension $(n \mid m)$. The symbol $p(A)$ denotes the parity of the object $A$ : it is equal to 0 if $A$ is even, it is equal to 1 if $A$ is odd.

Here we consider supermanifolds in the sense of F. Berezin, B. Kostant and D. Leites (see $[13,3,14])$. 
Definition 1. A supermanifold $M$ of dimension $(n \mid m)$ is a pair $\left(M_{0}, \mathcal{A}_{M}\right)$, where $M_{0}$ is a $n$ dimensional smooth manifold and where $\mathcal{A}_{M}$ is a sheaf of superfunctions, i.e., a sheaf of superalgebras such that for all $p \in M_{0}$, there is an open neighbourhood $U \ni p$ together with an isomorphism

$$
\Phi_{U}:\left.\left.\mathcal{A}_{M}\right|_{U} \stackrel{\sim}{\longrightarrow} C_{\mathbb{R}^{n}}^{\infty}\right|_{U^{\prime}} \otimes_{\mathbb{R}} \Lambda \mathbb{R}^{m},
$$

where $\Lambda \mathbb{R}^{m}$ denotes the exterior algebra of $\mathbb{R}^{m}$ and where $\left.C_{\mathbb{R}^{n}}^{\infty}\right|_{U^{\prime}}$ denotes the restriction to an open subset $U^{\prime}$ of the sheaf of smooth functions on $\mathbb{R}^{n}$.

The images by $\Phi_{U}^{-1}$ of the canonical coordinates of $\mathbb{R}^{n}$ (resp. of the canonical generators of $\Lambda \mathbb{R}^{m}$ ) give even superfunctions $x^{1}, \ldots, x^{n}$ (resp. odd superfunctions $x^{n+1}, \ldots, x^{n+m}$ ). We call $\left(U, \Phi_{U}\right)$ a local chart and $\left(x^{1}, \ldots, x^{n}, x^{n+1}, \ldots, x^{n+m}\right)$ graded coordinates on $M$.

If $\left(U, \Psi_{U}\right)$ is another local chart and if $\left(\bar{x}^{1}, \ldots, \bar{x}^{n}, \bar{x}^{n+1}, \ldots, \bar{x}^{n+m}\right)$ are corresponding graded coordinates, then the functions $\Phi_{U}\left(\bar{x}^{j}\right)$, which form the change of graded coordinates, will be denoted by $\bar{x}^{j}\left(x^{i}\right)$.

Definition 2. A morphism of supermanifolds $M \rightarrow N$ is a pair $\phi=\left(\phi_{0}, \phi^{*}\right)$, where $\phi_{0}: M_{0} \rightarrow$ $N_{0}$ is a smooth map and where $\phi^{*}: \mathcal{A}_{N} \rightarrow \mathcal{A}_{M}$ is a morphism of sheaves covering $\phi_{0}$, i.e., for every open subset $U \subset N_{0}, \phi^{*}$ gives a superalgebra morphism

$$
\phi^{*}(U): \mathcal{A}_{N}(U) \rightarrow \mathcal{A}_{M}\left(\phi_{0}^{-1}(U)\right)
$$

in a way compatible with the restriction maps. A diffeomorphism is a morphism such that $\phi_{0}$ is a diffeomorphism and such that $\phi^{*}$ is an isomorphism.

\subsection{Tensor densities}

The sheaf $\mathcal{F}_{\lambda, M}$ (or simply $\mathcal{F}_{\lambda}$ ) of $\lambda$-densities on $M$ is built from the Berezinian sheaf, whose formal definition can be found in [9]. For our purposes, it suffices to recall that, over an open set with graded coordinates $\left(x^{1}, \ldots, x^{n}, x^{n+1}, \ldots, x^{n+m}\right)$, a section of this sheaf, which we call a $\lambda$-density, is expressed formally as

$$
\phi|D x|^{\lambda},
$$

where $\phi$ is a local superfunction. Recall that, under a coordinate change $\bar{x}^{i}=\bar{x}^{i}\left(x^{j}\right)$, the element $|D x|^{\lambda}$ is multiplied by $|\operatorname{Ber} A|^{\lambda}$, where Ber denotes the Berezinian and where $A$ is the matrix corresponding to the change of coordinates, i.e., the matrix defined by

$$
A_{j}^{i}=\frac{\partial \bar{x}^{j}}{\partial x^{i}} .
$$

\subsection{Differential operators and symbols}

We denote by $\mathcal{D}_{\lambda, \mu, M}$ (or simply $\mathcal{D}_{\lambda, \mu}$ ) the sheaf of (finite-order) differential operators from $\lambda$-densities to $\mu$-densities. For an open subset $U$ of $M_{0}$, elements $D \in \mathcal{D}_{\lambda, \mu}(U)$ are local $\mathbb{R}$-linear maps $\mathcal{F}_{\lambda}(U) \rightarrow \mathcal{F}_{\mu}(U)$ for which there is an integer $k \in \mathbb{N}$ such that in any system of local graded coordinates $\left(x^{1}, \ldots, x^{n+m}\right)$, the restriction of $D$ reads

$$
\sum_{|\alpha| \leqslant k} D_{\alpha}\left(\frac{\partial}{\partial x^{1}}\right)^{\alpha_{1}} \cdots\left(\frac{\partial}{\partial x^{n+m}}\right)^{\alpha_{n+m}}
$$

where $\alpha$ is a multi-index, $|\alpha|=\sum_{i=1}^{n+m} \alpha_{i}, \alpha_{n+1}, \ldots, \alpha_{n+m}$ are in $\{0,1\}$ and $D_{\alpha}$ are local $\delta$-densities $(\delta=\mu-\lambda)$. The space $\mathcal{D}_{\lambda, \mu}(U)$ is thus filtered by the order of differential operators and we denote by $\mathcal{D}_{\lambda, \mu}^{k}(U)$ the space of differential operators of order at most $k$. 
As in the classical case, the highest order term of a differential operator behaves, under a change of graded coordinates, as a section of the sheaf of symbols, $\mathcal{S}_{\delta}^{k}=\mathcal{F}_{\delta} \otimes \vee^{k} \mathcal{T}_{M}$, where $\mathcal{T}_{M}$ denotes the tangent sheaf of $M$ and where $\vee$ denotes the supersymmetric product. This fact allows one to define the principal symbol operator $\sigma_{k}: \mathcal{D}_{\lambda, \mu}^{k} \rightarrow \mathcal{S}_{\delta}^{k}$. In graded coordinates,

$$
\sigma^{k}(D)=\sum_{|\alpha|=k} D_{\alpha}\left(\partial_{1}\right)^{\alpha_{1}} \vee \cdots \vee\left(\partial_{n+m}\right)^{\alpha_{n+m}}
$$

if $D$ is given by (1) and if $\partial_{1}, \ldots, \partial_{n+m}$ denotes the canonical basis of local supervector fields associated with the coordinate system $\left(x^{1}, \ldots, x^{n+m}\right)$. Moreover, we set $\mathcal{S}_{\delta}=\oplus_{k \geqslant 0} \mathcal{S}_{\delta}^{k}$.

\subsection{Superconnections and associated tensors}

A superconnection on $M$ is a morphism of sheaves of super vector spaces $\nabla: \mathcal{T}_{M} \otimes_{\mathbb{R}} \mathcal{T}_{M} \rightarrow \mathcal{T}_{M}$ such that for any open subset $U$ in $M_{0}$, for any $X, Y \in \mathcal{T}_{M}(U)$ and any $f \in \mathcal{A}_{M}(U)$,

$$
\nabla_{f X} Y=f \nabla_{X} Y \quad \text { while } \quad \nabla_{X} f Y=X(f) Y+(-1)^{p(X) p(f)} f \nabla_{X} Y
$$

where $\nabla_{X} Y$ stands for $\nabla\left(X \otimes_{\mathbb{R}} Y\right)$. Given graded coordinates $\left(x^{1}, \ldots, x^{n+m}\right)$ on $M$, a connection $\nabla$ reads

$$
\nabla_{X} Y=\left(X^{i} \partial_{i} Y^{k}+(-1)^{p(i)(p(Y)+p(j))} X^{i} Y^{j} \Gamma_{i j}^{k}\right) \partial_{k},
$$

where the so-called Christoffel symbols $\Gamma_{i j}^{k}$ of $\nabla$ are superfunctions with parity $p(i)+p(j)+p(k)$.

From a superconnection $\nabla$ on $M$, one defines its torsion tensor $\mathrm{T}^{\nabla}$ by

$$
\mathrm{T}^{\nabla}(X, Y)=\nabla_{X} Y-(-1)^{p(X) p(Y)} \nabla_{Y} X-[X, Y] .
$$

In graded coordinates, the vanishing of the torsion tensor of $\nabla$ translates into the supersymmetry of the Christoffel symbols in their low indices:

$$
\Gamma_{i j}^{k}=(-1)^{p(i) p(j)} \Gamma_{j i}^{k}
$$

We denote by $\mathcal{C}_{M}$ (or simply $\mathcal{C}$ ) the sheaf of torsion-free superconnections on $M$.

Remember also that from the curvature tensor $\mathrm{R}$ of $\nabla$, i.e.,

$$
\mathrm{R}(X, Y) Z=\nabla_{X} \nabla_{Y} Z-(-1)^{p(X) p(Y)} \nabla_{Y} \nabla_{X} Z-\nabla_{[X, Y]} Z,
$$

the super-Ricci tensor Ric and the tensor strR are defined as supertraces,

$$
\begin{aligned}
& \operatorname{Ric}(Z, Y)=\operatorname{str}\left(X \mapsto(-1)^{p(X)(p(Y)+p(Z))} \mathrm{R}(X, Y) Z\right), \\
& \operatorname{strR}(X, Y)=\operatorname{str}(Z \mapsto \mathrm{R}(X, Y) Z) .
\end{aligned}
$$

The super-Ricci tensor Ric and the tensor strR are then given in coordinates by the following formulas:

$$
\begin{aligned}
& \operatorname{Ric}(Z, Y)=(-1)^{p(i)(p(i)+p(Y)+p(Z))}\left(\mathrm{R}\left(\partial_{i}, Y\right) Z\right)^{i}, \\
& \operatorname{strR}(X, Y)=(-1)^{p(i)}\left(\mathrm{R}(X, Y) \partial_{i}\right)^{i}
\end{aligned}
$$

if $\mathrm{R}\left(\partial_{i}, Y\right) Z=\left(\mathrm{R}\left(\partial_{i}, Y\right) Z\right)^{j} \partial_{j}$ and $\mathrm{R}(X, Y) \partial_{i}=\left(\mathrm{R}(X, Y) \partial_{i}\right)^{j} \partial_{j}$.

Remark 1. Our tensor strR, the supertrace of the curvature, appears for instance in [9, Proposition 3.4]. 


\subsection{Projective equivalence of superconnections}

Definition 3. Two torsion-free superconnections $\nabla, \nabla^{\prime}$ are projectively equivalent if there is a super 1-form $\alpha$ on $M$ such that

$$
\nabla_{X}^{\prime} Y=\nabla_{X} Y+\alpha(X) Y+(-1)^{p(X) p(Y)} \alpha(Y) X .
$$

An equivalence class for this relation is called a projective class.

Locally, the condition for two torsion-free superconnections $\nabla$ and $\nabla^{\prime}$ to be projectively equivalent is expressed as $\Pi_{i j}^{k}=\Pi_{i j}^{\prime k}$, where

$$
\Pi_{i j}^{k}=\Gamma_{i j}^{k}-\frac{1}{n-m+1}\left(\Gamma_{i s}^{s} \delta_{j}^{k}(-1)^{p(s)}+\Gamma_{j s}^{s} \delta_{i}^{k}(-1)^{p(i) p(j)+p(s)}\right) .
$$

Obviously, this characterization fails when the superdimension $n-m$ is equal to -1 (in this case, the quantities $\Pi_{i j}^{k}$ cannot be defined). We believe that a detailed study of projective superconnections could provide a geometric meaning for this singular situation, but this is yet to be done.

\subsection{Problem setting}

A quantization on $M$ is an isomorphism $Q_{M}$ of sheaves of vector spaces, from the sheaf of symbols $\mathcal{S}_{\delta, M}$ to the sheaf of differential operators $\mathcal{D}_{\lambda, \mu, M}$ such that for any $k \in \mathbb{N}$ and any section $S$ of $\mathcal{S}_{\delta, M}^{k}$,

$$
\sigma^{k}\left(Q_{M}(S)\right)=S .
$$

Roughly speaking, a natural quantization is a quantization which depends on a torsion-free superconnection and commutes with the action of superdiffeomorphisms. More precisely, a natural quantization is a collection of morphisms (defined for every supermanifold $M$ )

$$
Q_{M}: \mathcal{C}_{M} \times \mathcal{S}_{\delta, M} \rightarrow \mathcal{D}_{\lambda, \mu, M}
$$

such that

- for any section $\nabla$ of $\mathcal{C}_{M}, Q_{M}(\nabla)$ is a quantization;

- if $\phi: M \rightarrow N$ is a local diffeomorphism of supermanifolds, then, for any section $\nabla$ of $\mathcal{C}_{N}$ and any section $S$ of $\mathcal{S}_{\delta, N}$,

$$
Q_{M}\left(\phi^{*} \nabla\right)\left(\phi^{*} S\right)=\phi^{*}\left(Q_{N}(\nabla)(S)\right) .
$$

A quantization $Q_{M}$ is projectively invariant if one has $Q_{M}(\nabla)=Q_{M}\left(\nabla^{\prime}\right)$ whenever $\nabla$ and $\nabla^{\prime}$ are projectively equivalent torsion-free superconnections.

The existence of natural and projectively invariant quantization is related to the existence of a $\mathfrak{p g l}(n+1 \mid m)$-equivariant quantization in the sense of [15] in the flat situation.

Definition 4. When $n-m \neq-1$, we define the numbers

$$
\gamma_{2 k-l}=\frac{(n-m+2 k-l-(n-m+1) \delta)}{n-m+1} .
$$

In this case, a value of $\delta$ is said to be critical if there is $k, l \in \mathbb{N}$ such that $1 \leq l \leq k$ and $\gamma_{2 k-l}=0$. Notice that, in opposition to the classical situation, the value $\delta=0$ can be critical since $n-m$ can be negative. 
One of the results of [15] is then the following.

Theorem 1. If $n-m \neq-1$ and $\delta$ is not critical, there exists a unique $\mathfrak{p g l}(n+1 \mid m)$-equivariant quantization. If $n-m=-1$, there exists a one-parameter family of $\mathfrak{p g l}(n+1 \mid m)$-equivariant quantizations.

As in the classical context, the projective class of the canonical flat superconnection is preserved by the vector fields of $\mathfrak{p g l}(n+1 \mid m)$. Therefore, if we can construct a natural projectively invariant quantization for supermanifolds of dimension $(n \mid m)$, it will necessarily coincide with the projectively equivariant quantization constructed in [15] when $M=\mathbb{R}^{n \mid m}$ and $\nabla$ is the projectively flat superconnection.

\section{Thomas fiber bundle and connection}

As in the classical case, a projective class of torsion-free superconnections on a supermanifold defines a superconnection on the associated "Thomas bundle". This fact was pointed out in [6] and is briefly recalled here.

\subsection{Thomas fiber bundle}

From a supermanifold $M$ of dimension $(n \mid m)$, the associated supermanifold $\tilde{M}$ is constructed by adding an even coordinate $x^{0}$ to each coordinate system $\left(x^{1}, \ldots, x^{n+m}\right)$. Under a change of coordinates $\bar{x}^{i}=\bar{x}^{i}\left(x^{j}\right)$ on $M$, this extra coordinate transforms according to the rule $\bar{x}^{0}=$ $x^{0}+\log |\operatorname{Ber} A|$ where $A$ is the matrix corresponding to the change of coordinates.

By analogy with the classical situation, we set $\mathcal{E}=\partial_{0}$ and call it the Euler supervector field of $\tilde{M}$. The fact that $\mathcal{E}$ is well-defined is easily seen from the transformation law of the components of a supervector field $X$ under a change of coordinates $\bar{x}^{i}=\bar{x}^{i}\left(x^{j}\right)$, namely

$$
X^{i} \partial_{x^{i}}=X^{i} \frac{\partial \bar{x}^{j}}{\partial x^{i}} \partial_{\bar{x}^{j}}
$$

Densities on $M$ identify with some superfunctions on $\tilde{M}$. More precisely, we can associate with a $\lambda$-density expressed locally as $f=\phi|D x|^{\lambda}$ the superfunction $\tilde{f}$ given by

$$
\tilde{f}\left(x^{0}, x^{1}, \ldots, x^{n+m}\right)=\phi\left(x^{1}, \ldots, x^{n+m}\right) \exp \left(\lambda x^{0}\right) .
$$

It follows directly from the transformation law of densities that $\tilde{f}$ is well-defined. Moreover, it is $\lambda$-equivariant in the sense that

$$
\mathrm{L}_{\mathcal{E}} \tilde{f}=\lambda \tilde{f} .
$$

Conversely, from a $\lambda$-equivariant superfunction $\varphi$ on $\tilde{M}$, one defines a $\lambda$-density $\varphi_{0}|D x|^{\lambda}$ on $M$ by setting

$$
\varphi_{0}\left(x^{1}, \ldots, x^{n}\right)=\varphi\left(x^{0}, x^{1}, \ldots, x^{n}\right) \exp \left(-\lambda x^{0}\right)
$$

for an arbitrary $x^{0}$. Because of the equivariance property of $\varphi$, the derivative of $\varphi_{0}$ with respect to $x^{0}$ is zero and the density is well-defined. This way, we establish a one-to-one correspondence between $\lambda$-densities on $M$ and $\lambda$-equivariant superfunctions on $\tilde{M}$. 


\subsection{Thomas connection}

The quantities $\Pi_{i j}^{k}$ given by (2) define the so-called fundamental descriptive invariant of the projective class of $\nabla$. They can be used to construct the projectively invariant lift $\tilde{\nabla}$ of $\nabla$ to the supermanifold $\tilde{M}$. More precisely, $\tilde{\nabla}$ is defined as the torsion-free superconnection on $\tilde{M}$ with Christoffel symbols

$$
\begin{aligned}
& \tilde{\Gamma}_{i j}^{k}=\Pi_{i j}^{k}, \quad \tilde{\Gamma}_{0 \mathfrak{a}}^{\mathfrak{c}}=\tilde{\Gamma}_{\mathfrak{a} 0}^{\mathfrak{c}}=\frac{-\delta_{\mathfrak{a}}^{\mathfrak{c}}}{n-m+1}, \\
& \tilde{\Gamma}_{i j}^{0}=\frac{n-m+1}{n-m-1}\left(\partial_{q} \Pi_{i j}^{q}-\Pi_{q i}^{p} \Pi_{p j}^{q}\right)(-1)^{p(q)(p(q)+p(i)+p(j))},
\end{aligned}
$$

where $i, j, k$ ranges from 1 to $n+m$ while $\mathfrak{a}, \mathfrak{c}$ ranges from 0 to $n+m$. Besides the singular case $n-m=-1$ (in which the quantities $\Pi_{i j}^{k}$ themselves cannot be defined), one must also assume here that $n-m \neq 1$ in order to give sense to the above formulas. The latter hypothesis is the super analogue of the fact that M. Bordemann's construction fails for a 1-dimensional smooth manifold.

We shall now give a useful coordinate-free description of the lifted superconnection $\tilde{\nabla}$ in terms of the Euler supervector field of $\tilde{M}$ and horizontal lifts of supervector fields on $M$. Using our coordinate system $\left(x^{0}, x^{1}, \ldots, x^{n+m}\right)$ (cf. Section 3.1), we introduce the so-called horizontal lift to $\tilde{M}$ of a supervector field $X=X^{i} \partial_{i}$ on $M$ by setting

$$
X^{h}=-(-1)^{p(s)} X^{i} \Gamma_{i s}^{s} \partial_{0}+X^{i} \partial_{i} .
$$

The vector field $X^{h}$ is well-defined. Indeed, the derivative of the Berezinian of the matrix $A$ representing the change of coordinates can be computed in the following way [13]:

$$
\partial_{x^{i}}(\operatorname{Ber} A)=(\operatorname{Ber} A) \operatorname{str}\left(\left(\partial_{x^{i}} A\right) A^{-1}\right),
$$

where $\left(\partial_{x^{i}} A\right)_{l}^{k}=(-1)^{p(i) p(k)} \partial_{x^{i}} A_{l}^{k}$ and where

$$
\operatorname{str}(B)=\sum_{i=1}^{p+q}(-1)^{p(i)(p(B)+p(i))} B_{i}^{i}
$$

if $B \in \mathfrak{g l}(n \mid m)$. We can then easily conclude using the transformation law of the Christoffel symbols:

$$
\bar{\Gamma}_{i j}^{k}=(-1)^{p(t)(p(l)+p(j))}\left(-\frac{\partial x^{t}}{\partial \bar{x}^{i}} \frac{\partial x^{l}}{\partial \bar{x}^{j}} \frac{\partial^{2} \bar{x}^{k}}{\partial x^{t} \partial x^{l}}+\frac{\partial x^{t}}{\partial \bar{x}^{i}} \frac{\partial x^{l}}{\partial \bar{x}^{j}} \Gamma_{t l}^{r} \frac{\partial \bar{x}^{k}}{\partial x^{r}}\right) .
$$

Definition 5. We denote by $r$ the following multiple of a supersymmetric part of the Ricci tensor of $\nabla$ :

$$
\mathrm{r}(X, Y)=\frac{1}{2(n-m-1)}\left(\operatorname{Ric}(Y, X)+(-1)^{p(X) p(Y)} \operatorname{Ric}(X, Y)\right) .
$$

Finally, we are in position to give the coordinate-free description of the lifted superconnection $\tilde{\nabla}$. This description is useful in order to simplify computations in the sequel.

Proposition 1. With notations of (3), we have for any supervector fields $X, Y$ on $M$,

$$
\begin{aligned}
& \tilde{\nabla}_{X^{h}} Y^{h}=\left(\nabla_{X} Y\right)^{h}-\frac{1}{2} \operatorname{str} \widetilde{R(X, Y) \mathcal{E}}+(n-m+1) \widetilde{\mathrm{r}(X, Y) \mathcal{E}}, \\
& \tilde{\nabla}_{X^{h}} \mathcal{E}=\tilde{\nabla}_{\mathcal{E}} X^{h}=\frac{-1}{n-m+1} X^{h}, \quad \tilde{\nabla}_{\mathcal{E}} \mathcal{E}=\frac{-1}{n-m+1} \mathcal{E} .
\end{aligned}
$$

Proof. The result is obtained after long but straightforward computations.

The lifted connection $\tilde{\nabla}$ is associated in a natural way with the connection $\nabla$ on $M$. Moreover, $\tilde{\nabla}$ is such that $\mathrm{L}_{\mathcal{E}} \tilde{\nabla}=0$, where

$$
\mathrm{L}_{\mathcal{E}} \tilde{\nabla}(X, Y)=\left[\mathcal{E}, \tilde{\nabla}_{X} Y\right]-\tilde{\nabla}_{[\mathcal{E}, X]} Y-\tilde{\nabla}_{X}[\mathcal{E}, Y]
$$


This invariance is due to the invariance of $\mathcal{E}$, of the horizontal lifts and of the functions $\operatorname{str} \widetilde{R(X, Y)}$ and $\widehat{\mathrm{r}} \widetilde{(X, Y)}$.

\section{Lift of symbols}

Our goal in this section is to lift in a natural and projectively invariant way a symbol $S$ on $M$ to a tensor $\tilde{S}$ on $\tilde{M}$. To this aim, we define in a first step a horizontal lift of $S$ via the horizontal lift of supervector fields (5). In a second step, we define a map which transforms equivariant tensors on $\tilde{M}$ into symbols on $M$. We prove that the restriction of this map to the divergencefree tensors (with respect to $\tilde{\nabla}$ ) is a bijection. The natural and projectively invariant lift is then the inverse map of this "descent" application.

\subsection{Horizontal lift of symbols}

Since a symbol $S$ of degree $k$ on $M$ is locally a sum of terms of the form $\phi|D x|^{\delta} \otimes \partial_{i_{1}} \vee \cdots \vee \partial_{i_{k}}$, it suffices to define the horizontal lift on symbols of this form and to extend it by linearity.

Definition 6. The horizontal lift of a symbol $\phi|D x|^{\delta} \otimes \partial_{i_{1}} \vee \cdots \vee \partial_{i_{k}}$, denoted by $\left(\phi|D x|^{\delta} \otimes \partial_{i_{1}} \vee\right.$ $\left.\cdots \vee \partial_{i_{k}}\right)^{h}$, is given in our coordinate system on $\tilde{M}$ by the tensor $\tilde{f} \otimes \partial_{i_{1}}^{h} \vee \cdots \vee \partial_{i_{k}}^{h}$ if $f=\phi|D x|^{\delta}$.

We can easily observe that the horizontal lift of a symbol $S$ is $\delta$-equivariant, i.e. that $\mathrm{L}_{\mathcal{E}} S^{h}=\delta S^{h}$. In particular, the horizontal lift of a $\delta$-density on $M$ to a superfunction on $\tilde{M}$ coincides with the correspondence given in (3).

\subsection{Descent map}

Using the fact that a tensor of degree $k$ on $\tilde{M}$ can be locally decomposed in the basis $\partial_{1}^{h}, \ldots, \partial_{n+m}^{h}$, $\mathcal{E}$, it is enough to define the descent map on a tensor of the form

$$
S=\sum_{l=0}^{k} \sum_{i_{1}, \ldots, i_{k-l}} \varphi^{i_{1}, \ldots, i_{k-l}, 0, \ldots, 0} \otimes \partial_{i_{1}}^{h} \vee \cdots \vee \partial_{i_{k-l}}^{h} \vee \mathcal{E}^{l}
$$

In the sequel, we denote by $\mathcal{S}_{\tilde{M}}^{k, \delta}$ the sheaf of $\delta$-equivariant tensors of degree $k$ on $\tilde{M}$.

Proposition 2. The map $\Psi$ whose value on a section $S$ of $\mathcal{S}_{\tilde{M}}^{k, \delta}$ expressed as in (6) is given by

$$
\Psi(S)=\sum_{i_{1}, \ldots, i_{k}} \varphi_{0}^{i_{1}, \ldots, i_{k}}|D x|^{\delta} \otimes \partial_{i_{1}} \vee \cdots \vee \partial_{i_{k}}
$$

where the coefficient $\varphi_{0}^{i_{1}, \ldots, i_{k}}$ is given by (4), is well-defined.

Proof. We can easily see that the form of $\Psi$ is preserved under a change of coordinates $\bar{x}^{i}=$ $\bar{x}^{i}\left(x^{j}\right)$ on $M$. It is among other things due to the fact that

$$
\varphi^{i_{1}, \ldots, i_{k}}\left(\bar{x}^{0}, \bar{x}^{1}, \ldots, \bar{x}^{n}\right) \exp \left(-\delta \bar{x}^{0}\right)=\varphi_{0}^{i_{1}, \ldots, i_{k}}\left(x^{1}, \ldots, x^{n}\right)|\operatorname{Ber} A|^{-\delta}=\bar{\varphi}_{0}^{i_{1}, \ldots, i_{k}}\left(\bar{x}^{1}, \ldots, \bar{x}^{n}\right),
$$

where $A$ stands for the matrix of the change of graded coordinates on $M$.

It is possible to show that the map $\Psi$ is surjective exactly in the same way as in [1]. If $A_{k}$ is a symbol of degree $k$ on $M$, then the sections of $\mathcal{S}_{\tilde{M}}^{k, \delta}$ whose images by $\Psi$ are equal to $A_{k}$ are those of the form

$$
A_{k}^{h}+A_{k-1}^{h} \vee \mathcal{E}+\cdots+A_{0}^{h} \vee \mathcal{E}^{k},
$$

for some sections $A_{k-j}$ of $\mathcal{S}_{\delta}^{k-j}$ for $j=1, \ldots, k$. 


\subsection{Projectively invariant lift of symbols}

In the sequel, we denote by $d x^{1}, \ldots, d x^{n+m}$ the dual basis of the canonical basis of local supervector fields $\partial_{1}, \ldots, \partial_{n+m}$ on $M$. It means that $d x^{i}\left(\partial_{j}\right)=\delta_{j}^{i}$ for all $i, j$.

The covariant derivative with respect to $\nabla$ of a $\delta$-density $\phi|D x|^{\delta}$ in the direction of a supervector field $X$ is given in coordinates by

$$
\nabla_{X}\left(\phi|D x|^{\delta}\right)=\left(X . \phi-(-1)^{p(s)} \delta X^{i} \Gamma_{i s}^{s} \phi\right)|D x|^{\delta} .
$$

We can easily show that this formula is preserved under a change of coordinates and that it defines a covariant derivative such that

$$
\widetilde{\nabla_{\partial_{i}} \phi|D x|^{\delta}}=\partial_{i}^{h} \cdot\left(\widetilde{\phi|D x|^{\delta}}\right) \text {. }
$$

Definition 7. The interior product of a symbol $S=\phi|D x|^{\delta} \otimes \partial_{i_{1}} \vee \cdots \vee \partial_{i_{k}}$ by a super 1-form $\alpha=\alpha_{i} d x^{i}$ is defined by

$$
\mathrm{i}(\alpha)(S)=\sum_{j=1}^{k}(-1)^{p(\alpha)\left(p(\phi)+p\left(i_{1}\right)+\cdots+p\left(i_{j-1}\right)\right)} \phi|D x|^{\delta} \otimes \partial_{i_{1}} \vee \cdots \vee \stackrel{(j)}{\alpha_{i_{j}}} \vee \cdots \vee \partial_{i_{k}},
$$

where $\alpha_{i_{j}}$ replaces $\partial_{i_{j}}$.

The interior product by a covariant tensor of degree $l, \alpha^{1} \vee \cdots \vee \alpha^{l}$, is then equal to $\mathrm{i}\left(\alpha^{1}\right) \circ$ $\cdots \circ \mathrm{i}\left(\alpha^{l}\right)$.

Definition 8. The covariant derivative with respect to $\nabla$ of a symbol $S=\phi|D x|^{\delta} \otimes \partial_{i_{1}} \vee \cdots \vee \partial_{i_{k}}$ in the direction of a supervector field $X$ is defined by

$$
\begin{aligned}
\nabla_{X}(S)= & \nabla_{X}\left(\phi|D x|^{\delta}\right) \otimes \partial_{i_{1}} \vee \cdots \vee \partial_{i_{k}} \\
& +\sum_{j=1}^{k}(-1)^{p(X)\left(p(\phi)+p\left(i_{1}\right)+\cdots+p\left(i_{j-1}\right)\right)} \phi|D x|^{\delta} \otimes \partial_{i_{1}} \vee \cdots \vee \nabla_{X}^{(j)} \partial_{i_{j}} \vee \cdots \vee \partial_{i_{k}} .
\end{aligned}
$$

Definition 9. The operator of divergence with respect to $\nabla$ is the map

$$
\operatorname{Div}: \mathcal{S}_{\delta} \rightarrow \mathcal{S}_{\delta}: S \mapsto \sum_{j=1}^{n+m}(-1)^{p(j)} \mathrm{i}\left(d x^{j}\right) \nabla_{\partial_{j}} S .
$$

We can easily check that this definition does not depend on the chosen coordinate system.

Proposition 3. If $j \in \mathbb{N}, l \in \mathbb{N}$ and if $A \in \mathcal{S}_{\delta}^{j}(M)$, then we have

$$
\widetilde{\operatorname{Div}}\left(A^{h} \vee \mathcal{E}^{l}\right)=(\operatorname{Div} A)^{h} \vee \mathcal{E}^{l}+2(n-m+1)(\mathrm{i}(\mathrm{r}) A)^{h} \vee \mathcal{E}^{l+1}-l \gamma_{2 j+l} A^{h} \vee \mathcal{E}^{l-1},
$$

where $\widetilde{\mathrm{Div}}$ stands for the divergence operator with respect to the lifted superconnection $\tilde{\nabla}$ on $\tilde{M}$ and $\mathrm{r}$ is the tensor introduced in Definition 5.

Proof. The proof is exactly the same as in [1].

If $\delta$ is not critical, the restriction of $\Psi$ to the divergence-free tensors with respect to $\tilde{\nabla}$ is thus a bijection. Indeed, the condition of zero divergence allows to fix the symbols $A_{k-j}$ in (7). These symbols are given by the following equations (for $0<l<k$ ):

$$
\begin{aligned}
& A_{k-1}=\frac{1}{\gamma_{2 k-1}} \operatorname{Div} A_{k}, \\
& A_{k-(l+1)}=\frac{1}{(l+1)\left(\gamma_{2 k-(l+1)}\right)}\left(\operatorname{Div} A_{k-l}+2(n-m+1) \mathrm{i}(\mathrm{r}) A_{k-(l-1)}\right) .
\end{aligned}
$$


Finally, the lift of a symbol $S$, denoted by $\tilde{S}$, is obtained by applying to $S$ the inverse of this bijection. This lift is natural thanks to the naturality of the condition linked to the divergence operator. The lift is also projectively invariant thanks to the fact that this condition depends only on $\tilde{\nabla}$.

\section{Construction of the projectively invariant quantization}

Definition 10. If $T$ is a supersymmetric covariant tensor of degree $l$ with values in $\lambda$-densities, $\nabla_{s} T$ is the supersymmetric covariant tensor of degree $l+1$ with values in the $\lambda$-densities defined in the following way:

$$
\begin{gathered}
\left(\nabla_{s} T\right)\left(X_{1}, \ldots, X_{l+1}\right)=\sum_{\sigma \in S_{l+1}}(-1)^{\epsilon_{l+1}+p(T) p\left(X_{\sigma(1)}\right)}\left(\nabla_{X_{\sigma(1)}}\left(T\left(X_{\sigma(2)}, \ldots, X_{\sigma(l+1)}\right)\right)\right. \\
\left.-\sum_{j=2}^{l+1}(-1)^{p\left(X_{\sigma(1)}\right)\left(p\left(X_{\sigma(2)}\right)+\cdots+p\left(X_{\sigma(j-1)}\right)\right)} T\left(X_{\sigma(2)}, \ldots, \nabla_{X_{\sigma(1)}} X_{\sigma(j)}, \ldots, X_{\sigma(l+1)}\right)\right),
\end{gathered}
$$

where $X_{1}, \ldots, X_{l+1}$ are supervector fields and where $\epsilon_{l+1}$ is the sign of the permutation $\sigma^{\prime}$ induced by $\sigma$ on the ordered subset of all odd elements among $X_{1}, \ldots, X_{l+1}$.

Definition 11. If $\varphi X_{1} \vee \cdots \vee X_{k}$ is a supersymmetric contravariant tensor of degree $k$ and if $\psi \alpha_{1} \vee \cdots \vee \alpha_{k}$ is a supersymmetric covariant tensor of degree $k$, then we set

$$
\begin{aligned}
& \left\langle\varphi X_{1} \vee \cdots \vee X_{k}, \psi \alpha_{1} \vee \cdots \vee \alpha_{k}\right\rangle \\
& \quad=\varphi \psi(-1)^{p(\psi)\left(p\left(X_{1}\right)+\cdots+p\left(X_{k}\right)\right)} \mathrm{i}\left(X_{1}\right) \cdots \mathrm{i}\left(X_{k}\right)\left(\alpha_{1} \vee \cdots \vee \alpha_{k}\right),
\end{aligned}
$$

where the interior product $\mathrm{i}$ is defined in the same way as in Definition 7 . One extends this operation by bilinearity to arbitrary supersymmetric tensors of degree $k$.

\subsection{The main result}

In this section, we give an explicit formula for the natural and projectively invariant quantization.

Theorem 2. If $n-m \neq \pm 1$ and $\delta$ is not a critical value, then the collection of maps $Q_{M}$ : $\mathcal{C} \times \mathcal{S}_{\delta} \rightarrow \mathcal{D}_{\lambda, \mu}$ given by

$$
\left(Q_{M}(\nabla, S)(f)\right)^{\sim}=\left\langle\tilde{S}, \tilde{\nabla}_{s}^{k} \tilde{f}\right\rangle,
$$

defines a projectively invariant natural quantization for supermanifolds of dimension $(n \mid m)$.

Proof. The proof goes exactly in the same way as in [1]. First, formula (8) is well-defined: indeed, the right-hand side is $\mu$-equivariant because of the invariance of $\tilde{\nabla}$, of the $\delta$-equivariance of $\tilde{S}$ and the $\lambda$-equivariance of $\tilde{f}$.

Next, (8) defines obviously a natural and projectively invariant quantization: this fact is mainly due to the naturality and the projective invariance of the lift $\tilde{S}$. The quantization preserves the principal symbol for the same reasons as in [1].

Remark 2. When $n-m \neq \pm 1, M=\mathbb{R}^{n \mid m}$ and $\nabla=\nabla_{0}$, formula (8) recovers the unique $\mathfrak{p g l}(n+1 \mid m)$-equivariant quantization found in [15]. It is interesting to notice the problem there was solved without any hypothesis on the superdimension. 
Remark 3. When $n-m \neq-1,1,-2,-4$ and $\delta=0$, formula (8) coincides with the canonical differential operator associated by J. George [6, Theorem 3.6] with a symbol of degree two and a projective class of superconnections. In particular, when $\lambda=\mu=0$, our formula recovers the projective Laplacian of [6, Theorem 3.2]. Notice that J. George does not have any formula for $n-m \in\{-2,-4\}$ while we do in general (those additional conditions appear only for particular values of the shift $\delta$ ). Actually, the methods used are different. The conditions $n-m \neq-1,1,-2$ in his work come from the construction, given a projective class on $M$, of the quantities $\tilde{\Pi}_{\mathfrak{b c}}^{\mathfrak{a}}$ associated with the corresponding Thomas connection while the condition $n-m \neq-4$ is due to the use of the projective Laplacian on $\tilde{M}$ (see [6, Theorem 3.2] for details). This being said, the relation between projectively invariant quantization and J. George's work is still unclear: one can wonder how the procedures are linked in the general case $\delta \neq 0$.

\section{The case $n-m=1$}

As it was already noticed in Section 3.2, the hypothesis $n-m \neq 1$ is the analogue in the super context of the fact that M. Bordemann's method [1] does not apply for 1-dimensional smooth manifolds.

Actually, the problem of natural and projectively invariant quantization on 1-dimensional smooth manifolds turns out to be very peculiar. In this case, it is easily shown that the difference between any two torsion-free linear connections can be expressed as $\alpha \vee$ id for some 1-form $\alpha$. Consequently, all torsion-free linear connections are projectively equivalent, and the quest for a natural projectively invariant quantization amounts to the quest for a natural bijection from symbols to differential operators. As it is well-known (it is for instance a consequence of [4, Theorem 3]), such a natural bijection does not exist. Notice that for symbols of order two, the theory of natural operators [8] imposes for a natural projectively invariant quantization to be of the form

$$
\left.Q(\nabla, S)(f)=\left\langle S, \nabla^{2} f\right\rangle+a\langle\operatorname{Div} S, \nabla f\rangle+b\left\langle\operatorname{Div}^{2} S, f\right\rangle+c\langle\text { i(Ric }) S, f\right\rangle,
$$

where $a, b, c \in \mathbb{R}$. The condition of projective invariance yields a system of equations for $a, b, c$ which admits no solution in dimension $n=1$ (cf. [2]).

If we make the assumption that a natural projectively invariant quantization must write under the form (9), with all objects being replaced by their super analogues, then the system of equations provided by the condition of projective invariance has no solutions when $n-m=1$. Therefore, unless there are more natural operators for supermanifolds than the superizations of the classical ones, a natural projectively invariant quantization does not exist in this case.

\section{The case $n-m=-1$}

In [15], P. Mathonet and F. Radoux were able to build a $\mathfrak{p g l}(n+1 \mid m)$-equivariant quantization without any hypothesis on the superdimension. Nevertheless, the case $n-m=-1$ required an ad-hoc construction because of the peculiarities of the Lie superalgebra $\mathfrak{p g l}(n+1 \mid n+1)$.

In our case, the problem lies in the very definition of the quantities $\Pi_{j k}^{i}$ used in the construction of the connection $\tilde{\nabla}$ on $\tilde{M}$ associated with a projective class of connections on $M$. The manifold $\tilde{M}$ is thus unhelpful here.

This being said, it can be checked by hand that the formula

$$
Q(\nabla, S)(f)=\langle S, \nabla f\rangle+t\langle\operatorname{Div} S, f\rangle
$$

defines a 1-parameter family of natural projectively invariant quantization for symbols of order one. This result agrees with the phenomenon observed in [15]. Also, for symbols of order two, 
the formula

$$
Q(\nabla, S)(f)=\left\langle S, \nabla^{2} f\right\rangle+\langle\operatorname{Div} S, \nabla f\rangle
$$

turns out to be projectively invariant. We conjecture that similar formulas can be obtained for higher order symbols and that a natural projectively invariant quantization exists when $n-m=-1$.

\section{Acknowledgements}

It is a pleasure to thank P. Mathonet for fruitful discussions. We also thank the referees for suggestions leading to great improvements of the original paper. Finally, F. Radoux thanks the Belgian FNRS for his research fellowship.

\section{References}

[1] Bordemann M., Sur l'existence d'une prescription d'ordre naturelle projectivement invariante, math.DG/0208171.

[2] Bouarroudj S., Projectively equivariant quantization map, Lett. Math. Phys. 51 (2000), 265-274, math.DG/0003054.

[3] Deligne P., Etingof P., Freed D.S., Jeffrey L.C., Kazhdan D., Morgan J.W., Morrison D.R., Witten E. (Editors), Quantum fields and strings: a course for mathematicians, Vols. 1, 2, American Mathematical Society, Providence, RI, 1999.

[4] Gargoubi Kh., Ovsienko V., Modules of differential operators on the line, Funct. Anal. Appl. 35 (2001), no. $1,13-18$.

[5] Dirac P.A.M., The fundamental equations of quantum mechanics, Proc. Roy. Soc. London 109 (1925), 642-653.

[6] George J., Projective connections and Schwarzian derivatives for supermanifolds, and Batalin-Vilkovisky operators, arXiv:0909.5419.

[7] Kirillov A.A., Geometric quantization, Current Problems in Mathematics. Fundamental Directions, Vol. 4, Itogi Nauki i Tekhniki, Akad. Nauk SSSR, Vsesoyuz. Inst. Nauchn. i Tekhn. Inform., Moscow, 1985, 141-178.

[8] Kolář I., Michor P.W., Slovák J., Natural operations in differential geometry, Springer-Verlag, Berlin, 1993.

[9] Kosmann-Schwarzbach Y., Monterde J., Divergence operators and odd Poisson brackets, Ann. Inst. Fourier (Grenoble) $\mathbf{5 2}$ (2002), 419-456, math.QA/0002209.

[10] Lecomte P.B.A., Ovsienko V.Yu., Projectively equivariant symbol calculus, Lett. Math. Phys. 49 (1999), 173-196, math.DG/9809061.

[11] Lecomte P.B.A., Ovsienko V.Yu., Cohomology of the vector fields lie algebra and modules of differential operators on a smooth manifold, Compositio Math. 124 (2000), 95-110, math.DG/9905058.

[12] Lecomte P.B.A., Towards projectively equivariant quantization, Progr. Theoret. Phys. Suppl. (2001), no. 144, $125-132$.

[13] Leĭtes D.A., Introduction to the theory of supermanifolds, Russ. Math. Surveys 35 (1980), no. 1, 1-64.

[14] Manin Yu.I., Gauge field theory and complex geometry, 2nd ed., Grundlehren der Mathematischen Wissenschaften, Vol. 289, Springer-Verlag, Berlin, 1997.

[15] Mathonet P., Radoux F., Projectively equivariant quantizations over the superspace $\mathbb{R}^{p \mid q}$, arXiv:1003.3320.

[16] Woodhouse N.M.J., Geometric quantization, 2nd ed., Oxford Mathematical Monographs, Oxford Science Publications, The Clarendon Press, Oxford University Press, New York, 1992. 